\title{
Hyperglycaemia-induced pro-inflammatory responses by retinal Müller glia are regulated by the receptor for advanced glycation end-products (RAGE)
}

\author{
H. Zong • M. Ward • A. Madden • P. H. Yong • \\ G. A. Limb - T. M. Curtis • A. W. Stitt
}

Received: 24 June 2010 / Accepted: 9 August 2010/Published online: 12 September 2010

(C) Springer-Verlag 2010

\begin{abstract}
Aims/hypothesis Up-regulation of the receptor for AGEs (RAGE) and its ligands in diabetes has been observed in various tissues. Here, we sought to determine levels of RAGE and one of its most important ligands, S100B, in diabetic retina, and to investigate the regulatory role of S100B and RAGE in Müller glia.

Methods Streptozotocin-diabetes was induced in SpragueDawley rats. RAGE, S100B and glial fibrillary acidic protein (GFAP) were detected in retinal cryosections. In parallel, the human retinal Müller cell line, MIO-M1, was maintained in normal glucose $(5.5 \mathrm{mmol} / \mathrm{l})$ or high glucose (25 mmol/l). RAGE knockdown was achieved using small interfering RNA (siRNA), while soluble RAGE was used as a competitive inhibitor of RAGE ligand binding. RAGE, S100B and cytokines were detected using quantitative RTPCR, western blotting, cytokine protein arrays or ELISA. Activation of mitogen-activated protein kinase (MAPK) by RAGE was determined by western blotting.

Results Compared with non-diabetic controls, RAGE and S100B were significantly elevated in the diabetic retina with apparent localisation in the Müller glia, occurring concomitantly with upregulation of GFAP. Exposure of
\end{abstract}

Electronic supplementary material The online version of this article (doi:10.1007/s00125-010-1900-z) contains supplementary material, which is available to authorised users.

H. Zong · M. Ward · A. Madden • P. H. Yong · T. M. Curtis •

A. W. Stitt $(\square)$

Centre for Vision and Vascular Science, Queen's University

Belfast, Royal Victoria Hospital,

Belfast BT12 6BA Northern Ireland, UK

e-mail: a.stitt@qub.ac.uk

G. A. Limb

Institute of Ophthalmology, University College London,

London, UK
MIO-M1 cells to high glucose induced increased production of RAGE and S100B. RAGE signalling via MAPK pathway was linked to cytokine production. Blockade of RAGE prevented cytokine responses induced by high glucose and S100B in Müller glia.

Conclusions/interpretation Hyperglycaemia in vivo and in vitro exposure to high glucose induce upregulation of RAGE and its ligands, leading to RAGE signalling, which links to pro-inflammatory responses by retinal Müller glia. These data shed light on the potential clinical application of RAGE blockade to inhibit the progression of diabetic retinopathy.

Keywords Cytokine - Diabetic retinopathy - Inflammation · MAPK $\cdot$ Müller glia $\cdot$ RAGE $\cdot$ S100B
Abbreviations
GFAP Glial fibrillary acidic protein
GST Glutathione $S$-transferase
HMGB1 High mobility group box 1 protein
MAPK Mitogen-activated protein kinase
MCP-1 Monocyte chemotactic protein-1
MEK MAPK/ERK kinase
RAGE Receptor for AGEs
siRNA Small interfering RNA
sRAGE Soluble receptor for AGEs
VEGF Vascular endothelial growth factor

\section{Introduction}

During diabetes, retinal cells are exposed to sustained hyperglycaemia-related biochemical stress, leading to dysfunctional responses in a range of cell types including the 
neurons, glia and the supporting microvasculature. Diabetic retinopathy is best recognised as a vasodegenerative disease with impaired blood flow regulation, increased vasopermeability, capillary basement membrane thickening, microaneurysm formation, microvascular cell death and eventually widespread non-perfusion and ischaemia of the inner retina [1]. These diabetes-related changes often occur in unison with neuronal and glial dysfunction. In particular the retinal Müller glial cell is now viewed as playing an important role in the initiation and progression of diabetic retinopathy. These macroglia have a unique role in the architecture and physiology of the retina and demonstrate considerable dysfunction during diabetes [2]. During early diabetes, Müller glia show a range of responses such as increased levels of glial fibrillary acidic protein (GFAP) [3] and synthesis of glutamate, which may contribute to excitotoxicity in retinal neurons [4]. In the later stages of diabetes, Müller glia dysfunction can directly drive retinal neovascularisation [5] and contribute to oedema by cytoplasmic swelling [6].

While the precise pathogenesis of diabetic retinopathy remains incompletely understood, inflammation and related processes are now thought to contribute to neuronal, glial and microvascular lesions. The aberrant levels of proinflammatory cytokines within the neural retina and upregulation of adhesion molecules on the microvasculature leading to leukostatic responses have been linked to the formation of acellular capillary formation [7]. Global mRNA expression profiling has also highlighted altered expression of cytokines and interrelated pathways, not only in the retinal vessels [8], but also in the neuroglia [9]. A complex milieu of dysregulated pro-inflammatory factors occurs in diabetic retina [10], and while retinal microglia and infiltrating monocytic cells probably make an important contribution [11], there is also strong evidence that Müller glia show inflammation-linked responses when exposed to the diabetic milieu [12].

Many macro- and microvascular diabetic complications show pro-inflammatory aetiology, one component of which is activation of the receptor for AGEs (RAGE) [13]. RAGE is a pattern recognition receptor that binds to several ligands including S100/calgranulins, amphoterin (high mobility group box 1 protein [HMGB1]), amyloid- $\beta$, CD11b/CD18 and AGEs [14]. RAGE is a member of the immunoglobulin superfamily transmembrane protein, composed of one IgV-like domain, two tandem $\mathrm{C}$ type domains, a transmembrane region and a cytosolic tail [15]. Various signalling pathways can be induced by RAGE and its ligands, such as mitogen-activated protein kinases (MAPKs) (including extracellular signal-regulated kinases 1 and 2, and p38), Janus kinase/signal transducers and activators of transcription, Rho GTPases and nuclear factor KB [16-19]. By regulating these pathways, RAGE plays an important role in inappropriate innate immune responses during developments associated with Alzheimer's disease, atherosclerosis, pulmonary disorders and diabetic complications [20].

RAGE production in the retina has been predominantly localised to glia in the inner retina and it is established that RAGE is elevated in diabetic conditions [21, 22]. RAGE ligands have also been demonstrated in the retina, especially various AGE adducts that accumulate in diabetes [23, 24]. Other RAGE ligands including S100/calgranulins and HMGB1 are evident in the vitreous and pre-retinal membranes of eyes with proliferative diabetic retinopathy and proliferative vitreoretinopathy [25]. It has been reported that hyperglycaemia increases RAGE, S100A8, S100A12 and HMGB1 levels in human aortic endothelial cells, a response that can be normalised by the superoxide dismutase mimetic manganese(III) tetrakis (4-benzoic acid)porphyrin chloride (MnTBAP) [26]. However, such responses have not been previously shown in diabetic retina. Moreover, the biological consequences of upregulation of RAGE and its ligands in retinal cells needs to be clarified, since there is clear potential for activation of this receptor in the context of diabetic retinopathy.

In the current investigation, we examined RAGE and its primary ligand S100B in the diabetic retina and in Müller glia exposed to high glucose conditions. The response of Müller glia to hyperglycaemia or RAGE activation was studied. Our data suggest a key role for this receptor in hyperglycaemia-induced activation of Müller glia, a finding that has implications for further deciphering the complex pathogenesis of diabetic retinopathy.

\section{Methods}

Diabetic animal model Animal studies were conducted in accordance with British Home Office regulations. Diabetes was induced in male Sprague-Dawley rats (150-200 g, 6 weeks old; Harlan Laboratories, Horst, the Netherlands) by a single intravenous injection of streptozotocin $(45 \mathrm{mg} /$ $\mathrm{kg}$ in $0.1 \mathrm{~mol} / 1$ citrate buffer, $n=10)$. A separate group received citrate buffer alone $(n=10)$. Animals with blood glucose levels $>15 \mathrm{mmol} / 1$ at 1 week after streptozotocininjection were included in the study. Water intake and blood glucose were monitored monthly and animals received insulin injections three times per week (3-4 IU ultralente insulin; Eli Lilly, Basingstoke, UK) to promote weight gain and limit hyperglycaemia. At 4 months after diabetes induction, animals were re-weighed and blood was sampled monthly for measurement of plasma glucose concentrations and per cent total glycated haemoglobin (Helena Biosciences, Gateshead, UK). These variables were also measured immediately prior to experimentation. 
Preparation of retinal cryosections Eyes were enucleated and fixed for $1 \mathrm{~h}$ in $4 \%$ (wt/vol.) paraformaldehyde in PBS at room temperature. After fixation, the posterior eye cups were cryoprotected and embedded in OCT (Sakura Finetek, Torrance, CA, USA), and 16 to $30 \mu \mathrm{m}$ cryosections were prepared and mounted onto slides.

Immunofluorescence Cryosections were rinsed in PBS and blocked for $1 \mathrm{~h}$ in blocking buffer (10\% [vol./vol.] normal goat serum, $0.3 \%$ [vol./vol.] Triton X-100 in PBS) at room temperature. The slides were then incubated for $24 \mathrm{~h}$ with S100B (Abcam, Cambridge, UK) and RAGE (Millipore, Dundee, UK) or GFAP (Dako, Cambridgeshire, UK) antibodies diluted in blocking buffer at $4^{\circ} \mathrm{C}$. Following removal of the primary antibodies, slides were extensively washed and incubated for $1 \mathrm{~h}$ in fluorescent-conjugated secondary antibodies (Alexa Fluor 488 and 568; Invitrogen, Paisley, UK) at room temperature. After several washing steps in PBS, cell nuclei were counterstained with $10 \mu \mathrm{g} / \mathrm{ml}$ propidium iodide (Sigma-Aldrich, Poole, UK) in PBS containing $10 \mu \mathrm{g} / \mathrm{ml}$ RNase A (Invitrogen, Paisley, UK). After further washing, the slides were mounted in Vectashield anti-fade mounting medium (Dako, Cambridgeshire, UK) and visualised using a confocal system (TE-2000 C1; Nikon, Surrey, UK).

For immunofluorescence using cultured cells, cells growing on coverslips were fixed in $4 \%$ (wt/vol.) paraformaldehyde for $1 \mathrm{~h}$ at room temperature, followed by permeabilisation using $0.2 \%$ (vol./vol.) Triton X-100/5\% (wt/vol.) BSA/PBS for 5 min on ice. The fixed cells were then blocked in $5 \%(\mathrm{wt} / \mathrm{vol}$.) BSA/PBS for $1 \mathrm{~h}$ at room temperature. Immunofluorescence in the cultured cells was determined using RAGE, $\beta$-actin (Sigma-Aldrich, Poole, UK) or mouse normal IgG as described above.

Cell culture, treatments and transfections The human adult Müller glial cell line MIO-M1 [27] was cultured in DMEM containing $5.5 \mathrm{mmol} / \mathrm{l}$ glucose (Invitrogen, Paisley, UK), $10 \%$ (vol./vol.) FCS and $10 \mathrm{mg} / \mathrm{ml}$ primocin (InvivoGen, San Diego, CA, USA) in a humidified atmosphere of $5 \%$ (vol./vol.) $\mathrm{CO}_{2}$ at $37^{\circ} \mathrm{C}$. For experiments, MIO-M1 cells were grown in DMEM and 10\% (vol./vol.) FCS containing $5.5 \mathrm{mmol} / \mathrm{l}$ glucose (normal glucose) or $25 \mathrm{mmol} / \mathrm{l}$ glucose (high glucose) for up to 3 days, followed by fluorescence microscopy, RNA or protein extraction. An osmolality control was also used by supplementing $20 \mathrm{mmol} / \mathrm{l}$ mannitol in normal glucose-containing DMEM. MIO-M1 cells were maintained in DMEM and 2\% (vol./vol.) FCS containing normal glucose or high glucose for $24 \mathrm{~h}$ prior to S100B treatment.

For siRNA transfection, $1 \times 10^{6}$ cells were plated on $60 \mathrm{~mm}$ Petri dishes. After $24 \mathrm{~h}$, cells were transfected with scrambled or RAGE-specific siRNA (Ambion, Austin, TX,
USA) with Lipofectamine 2000 (Invitrogen, Paisley, UK) according to the manufacturer's instructions. S100B treatment was performed $48 \mathrm{~h}$ post transfection.

$R N A$ extraction and quantitative RT-PCR For quantitative PCR, primers for human $V E G F$ (also known as VEGFA), $\beta$ actin and $M C P-1$ (also known as CCL2) were designed using Primer 3 (http://frodo.wi.mit.edu/primer3/; last accessed 12 January 2009) (Electronic supplementary material [ESM] Table 1). The primers for human S100B and full length RAGE were used as described [28, 29]. Total RNA was extracted from MIO-M1 cells with TRI reagent (Sigma-Aldrich, Poole, UK) according to the manual. First strand cDNA was synthesised using $500 \mathrm{ng}$ of total RNA and Superscript III reverse transcriptase (Invitrogen, Paisley, UK). Quantitative PCR was performed and analysed using a kit (SYBR green PCR kit and LightCycler 480; Roche, Burgess Hill, UK).

Cytokine profile assay and ELISA MIO-M1 cells cultured in six-well plates were treated with RAGE ligands for $24 \mathrm{~h}$. The culture medium was collected and subjected to the cytokine profile assay (Randox, Crumlin, UK). The concentrations of cytokines were normalised to total cell numbers in the same well. Vascular endothelial growth factor (VEGF) and S100B levels were determined using a human VEGF ELISA kit (R\&D Systems, Minneapolis, MN, USA) and a human S100B ELISA kit (Abnova, Walnut, CA, USA) according to the manufacturers' instructions.

Western blotting MIO-M1 cell lysates were prepared with RIPA buffer $(50 \mathrm{mmol} / \mathrm{l}$ Tris $\cdot \mathrm{HCl}, \mathrm{pH} 7.5,150 \mathrm{mmol} / \mathrm{l} \mathrm{NaCl}, 1 \%$ [vol./vol.] Triton X-100, 0.1\% [wt/vol.] SDS, $1 \mathrm{mmol} / 1$ EDTA, $5 \mathrm{mmol} / \mathrm{l} \beta$-glycerophosphate, $1 \mathrm{mmol} / 1 \mathrm{Na}_{3} \mathrm{VO}_{4}$, $1 \mathrm{mmol} / 1 \mathrm{NaF}, 1 \times$ protease inhibitor cocktail [Roche, Burgess Hill, UK]) on ice for $30 \mathrm{~min}$. Total protein $(20 \mu \mathrm{g})$ was applied to a $10 \%$ SDS-PAGE gel, blotted to polyvinylidene fluoride membrane (Immobilon-FL; Millipore, Watford, UK) and probed overnight at $4{ }^{\circ} \mathrm{C}$ with rabbit monoclonal p44/42 or phospho-p44/42 (Thr202/Tyr204) antibody (Cell Signaling Technology, Boston, MA, USA) alongside mouse monoclonal $\beta$-actin antibody (Sigma), followed by incubation for $30 \mathrm{~min}$ at room temperature with IRDye 680 goat anti-rabbit and IRDye $800 \mathrm{CW}$ goat anti-mouse secondary antibodies (Li-COR, Lincoln, NE, USA). The membrane was then scanned and analysed using an infrared imaging system (Odyssey; Li-COR, Lincoln, NE, USA).

Soluble RAGE (sRAGE) preparation We cultured Eschericia coli carrying pGEX vector or pGEX-sRAGE overnight in Lennox broth (LB) at $37^{\circ} \mathrm{C}$. After 12 to $16 \mathrm{~h}, 2 \mathrm{ml}$ of the 
culture was transferred into $200 \mathrm{ml}$ of fresh LB and shaken for $3 \mathrm{~h}$ at $37^{\circ} \mathrm{C}$, followed by treatment with $0.2 \mathrm{mmol} / 1$ isopropyl $\beta$-D-1-thiogalactopyranoside (IPTG) for another $4 \mathrm{~h}$ at $37^{\circ} \mathrm{C}$. The $E$. coli were collected and lysed in Buffer A $(25 \mathrm{mmol} / \mathrm{l}$ Tris $\cdot \mathrm{HCl}, \mathrm{pH} 8.0,1 \mathrm{mg} / \mathrm{ml}$ lysozyme, $1 \mathrm{mg} / \mathrm{ml}$ DNase I, $100 \mathrm{mmol} / 1 \mathrm{NaCl}, 1 \mathrm{mmol} / \mathrm{l}$ DTT, $1 \mathrm{mmol} / 1 \mathrm{PMSF}$ ). Glutathione $S$-transferase (GST) protein containing bacterial lysates was reserved for further purification. For sRAGE, the pellet from the bacterial lysates was collected by centrifugation at $20,000 \times \mathrm{g}$ for $10 \mathrm{~min}$ at $4^{\circ} \mathrm{C}$ and washed with Buffer $\mathrm{B}(25 \mathrm{mmol} / \mathrm{l}$ Tris $\cdot \mathrm{HCl}, \mathrm{pH} 8.0,100 \mathrm{mmol} / \mathrm{l} \mathrm{NaCl}, 1 \mathrm{mmol} / \mathrm{l}$ DTT, $1 \mathrm{mmol} / 1 \mathrm{PMSF}, 1 \%$ [vol./vol.] Triton X-100) and Buffer C (25 mmol/1 Tris $\cdot \mathrm{HCl}, \mathrm{pH} 8.0,100 \mathrm{mmol} / 1 \mathrm{NaCl}, 1 \mathrm{mmol} / 1$ DTT, $1 \mathrm{mmol} / 1 \mathrm{PMSF}, 2 \mathrm{~mol} / \mathrm{l}$ urea). The pellet was then re-suspended in Buffer D $(50 \mathrm{mmol} / 1$ Tris $\cdot \mathrm{HCl}, \mathrm{pH} 8.0$, $10 \mathrm{mmol} / \mathrm{l} \mathrm{DTT,} 8 \mathrm{~mol} / \mathrm{l}$ urea). The aqueous fraction was dialysed with ingredient urea buffer $(25 \mathrm{mmol} / \mathrm{l}$ Tris $\cdot \mathrm{HCl}$, $\mathrm{pH} 8.0,100 \mathrm{mmol} / \mathrm{l} \mathrm{NaCl}$ and $1 \mathrm{mmol} / \mathrm{l}$ DTT; supplemented with $6,4,3,2,1$ and $0 \mathrm{~mol} / 1$ of urea, respectively) in Slide-A-Lyzer dialysis cassettes (Pierce, Rockford, IL, USA). GST-containing lysates and the dialysed samples were incubated with MagneGST beads (Promega, Southampton, UK) to purify GST and GSTsRAGE. GST or GST-sRAGE was recovered from the MagneGST beads by elution with $50 \mathrm{mmol} / 1$ glutathione and dialysed to remove glutathione.

Statistical analysis Results were expressed as mean $\pm \mathrm{SE}$. Statistical analyses were performed using Prism V4.02 (Graphpad Software, San Diego, CA, USA). Data were evaluated for normal distribution and then one-way ANOVA was conducted to compare overall treatment differences. Post-hoc multiple pair-wise comparisons were performed using Duncan's multiple range test.

\section{Results}

Diabetes characterisation in rats At 4 months of diabetes, glycated haemoglobin was elevated in the diabetic group compared with non-diabetic controls $(6.45 \% \pm 0.41$ vs $19.66 \% \pm 2.32 ; p<0.001)$. Body weight decreased in diabetic rats $(287 \pm 9.4 \mathrm{~g})$ compared with non-diabetic controls $(431 \pm 9.4 \mathrm{~g}, p<0.001)$.

RAGE is elevated in Müller glia in diabetic retina To investigate localisation of key elements of the RAGE axis in diabetic retinopathy, immunoreactivity of RAGE and S100B was determined. S100B was present in layers of the inner retina in a pattern indicative of astrocytic/Müller glia localisation. RAGE was more abundant throughout the neuropile and showed a pattern very similar to that previously reported by Barile et al. [21]. In diabetic retina, the levels of S100B and RAGE were markedly increased compared with non-diabetic retina. S100B was localised to the transretinal cytoplasmic processes characteristic of Müller glia (Fig. 1a). To confirm co-localisation with Müller glia, GFAP was used as an astrocyte/Müller glia marker. Co-localisation of S100B and GFAP was observed in diabetic retina only (Fig. 1b).

RAGE is increased in Müller glia exposed to high glucose To ascertain whether upregulation of RAGE in retinal Müller glia is linked to hyperglycaemia, MIO-M1 cells were cultured in normal glucose or high glucose for 3 days. RAGE protein was determined by immunofluorescence microscopy and the RAGE receptor was found to be present on the cell surface, with enhanced production following exposure to high glucose when compared with normal glucose controls (Fig. 2a, b). Interestingly, MIO-M1 culture in high glucose showed more pseudopodia, in which RAGE was highly abundant, suggesting that RAGE could be linked to polarisation of Müller glia. Western blot analysis also revealed high glucose-induced increase of RAGE, a response also demonstrated by GFAP-immunoreactivity (Fig. 2c). Quantitative RT-PCR was performed to detect mRNA expression levels of RAGE, GFAP and $S 100 B$, which were increased by 1.5 to 2 -fold in cells exposed to high glucose $(p<0.01$, Fig. 2d). Mannitol-supplemented normal glucose medium did not show elevated mRNA expression of $R A G E, S 100 B$ and GFAP (data not shown).

To verify the role of RAGE in high glucose-induced GFAP production in Müller glia, RAGE-specific siRNA (siRAGE) or scrambled control siRNA (scr) were transfected into MIO-M1 cells. An effective knockdown of RAGE was demonstrated (Fig. 2b-d). RAGE siRNA not only inhibited high glucose-mediated increase in RAGE protein and mRNA expression, but also prevented the induction of GFAP by high glucose exposure $(p<0.01$, Fig. 2c, d). $S 100 B$ overexpression in response to high glucose was unaltered by RAGE knockdown (Fig. 2d).

S100B is induced by hyperglycaemia In non-diabetic rat retina, ELISA analysis demonstrated that S100B protein was present at $\sim 30 \mathrm{pg} / \mu \mathrm{g}$ of total retinal protein. In the diabetic retina, a threefold increase of S100B protein was seen (Fig. 3a). S100B ELISA was also performed using the total protein extracted from MIO-M1 cells to determine whether Müller cells produce S100B and whether exposure of Müller glia to high glucose results in elevated S100B levels. We found that S100B protein was produced in MIOM1 cells at $\sim 6 \mathrm{pg} / \mu \mathrm{g}$ of total protein, with production increased by 3-, 3.5- and 4.2-fold upon 1, 2 and 3 days of high glucose treatment, respectively (Fig. 3b). 
Fig. 1 Enhanced RAGE and S100B levels in diabetic retina. Retinas were isolated from control or streptozotocin-induced diabetic rats and cryosections were stained (a) with rabbit polyclonal anti-S100B and mouse monoclonal anti-RAGE antibodies or (b) with rabbit polyclonal anti-S100B and mouse monoclonal anti-GFAP antibodies. ILM, inner limiting membrane; IPL, inner plexiform layer; INL, inner nuclear layer; ONL, outer nuclear layer; ELM, external limiting membrane. Scale bars, $50 \mu \mathrm{m}$ a
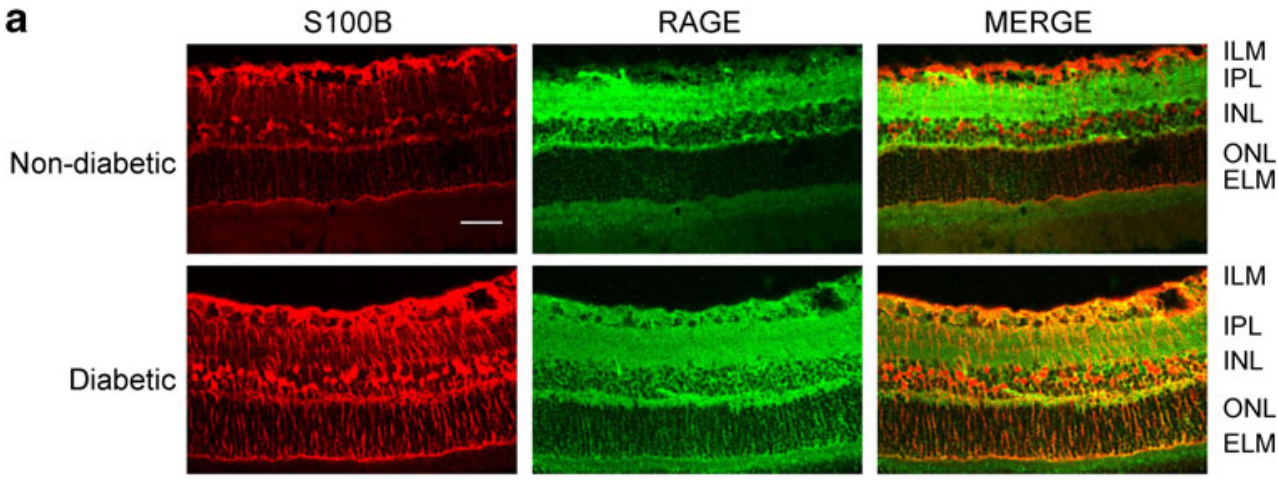

b
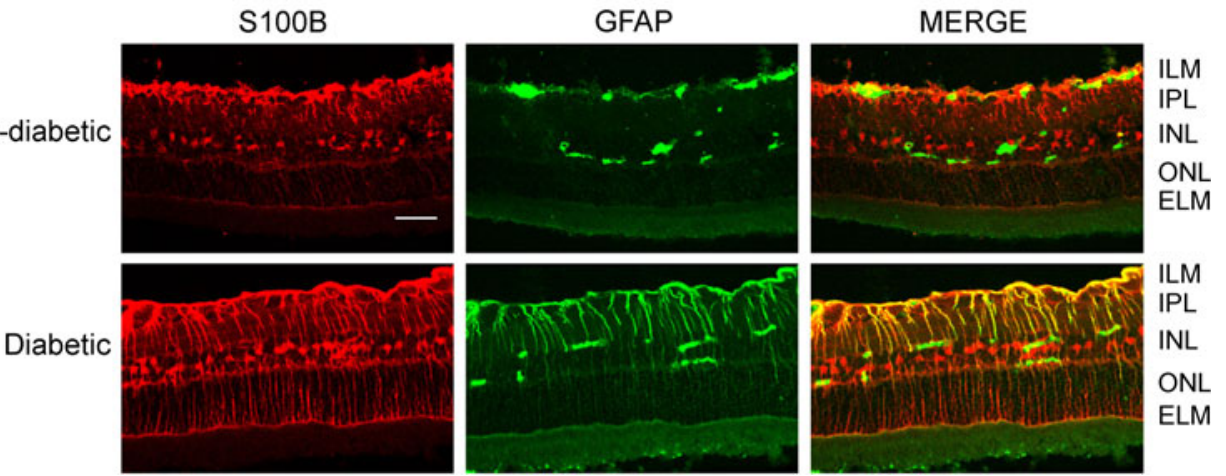

RAGE mediates activation of MAPK pathway in Müller glia To investigate the function of RAGE in Müller glia, activation of the MAPK pathway by S100B was determined. MIO-M1 cells were exposed to increasing concentrations of $\mathrm{S} 100 \mathrm{~B}$ for $30 \mathrm{~min}$. Western blot analysis for phosphorylated p44/42 MAPK and MAPK/ERK kinase (MEK) $1 / 2$ demonstrated that $\mathrm{S} 100 \mathrm{~B}$ at up to $200 \mathrm{nmol} / \mathrm{l} \mathrm{did}$ not alter activation of the MAPK pathway, but a dosedependent activation of MAPK was observed when cells were treated with over $500 \mathrm{nmol} / \mathrm{l} \mathrm{S100B}$ (Fig. 4a). MAPK activation by $\mathrm{S} 100 \mathrm{~B}$ was also appreciably accentuated by exposure to high glucose (Fig. 4b). The phosphorylation of p44/42 was also time-dependent. Exposure of MIO-M1 cells to S100B led to phosphorylation of p44/42 within $5 \mathrm{~min}$, which peaked at $15 \mathrm{~min}$ and subsequently tailed off by $120 \mathrm{~min}$ (Fig. 4c). U0126, the MEK1/2-specific inhibitor, was used to treat MIO-M1 cells prior to S100B treatment and abrogated activation of p44/42 by S100B/RAGE (Fig. 4d).

RAGE modulates cytokine production by Müller glia An important feature of Müller glial activation under diabetic and some other disease conditions is the release of proinflammatory cytokines $[12,30]$. MIO-M1 cells were treated with $1 \mu \mathrm{mol} / \mathrm{l}$ of $\mathrm{S} 100 \mathrm{~B}$ for $24 \mathrm{~h}$. Cell culture medium was collected to detect various pro-inflammatory cytokines including IL-2, IL-4, IL-6, IL-8, IL-10, VEGF, IFN- $\gamma$, TNF- $\alpha$, IL- $1 \alpha$, IL- $1 \beta$, monocyte chemotactic protein-1 (MCP-1) and EGF. In MIO-M1 culture medium, IL-2, IL-10 and IL-1 $\alpha$ were undetectable. However, IL-6, IL-8, VEGF and MCP-1 exhibited high abundance levels, which were significantly enhanced by $\mathrm{S} 100 \mathrm{~B}(p<0.001$; Fig. 5a). Although the basic levels of IFN- $\gamma$, TNF- $\alpha$, IL$1 \beta$ and EGF were relatively low $(<10 \mathrm{pg} / \mathrm{ml})$, their induction by $\mathrm{S} 100 \mathrm{~B}$ was also significant $(p<0.05$; Fig. 5a, b).

$V E G F$ and $M C P-1$ were chosen for further quantitative RT-PCR analysis, which revealed dose-dependent upregulation of these cytokines along with RAGE mRNA following exposure to micromolar levels of $\mathrm{S} 100 \mathrm{~B}$ (Fig. 5c). Inhibition of the MAPK pathway by U0126 prevented S100B-induced VEGF expression $(p<0.01$; Fig. 5d).

High glucose and $S 100 B$ promote cytokine expression in Müller cells via RAGE To further verify that exposure to high glucose is linked to levels of RAGE and subsequent release of cytokines, MIO-M1 cells were exposed to high glucose for increasing time-periods, and VEGF, MCP-1, $S 100 B$ and $R A G E$ mRNA levels determined. RAGE mRNA expression was slightly increased at $8 \mathrm{~h}$ of exposure to high glucose and significantly elevated at $24 \mathrm{~h}(p<0.05)$, peaking at $48 \mathrm{~h}(p<0.05$; Fig. 6$)$. In comparison, $S 100 B$ mRNA expression was not altered prior to the 24th hour of high glucose treatment, but was induced by about fourfold from $24 \mathrm{~h}(p<0.001$; Fig. 6). $V E G F$ and $M C P-1$ were also 
a

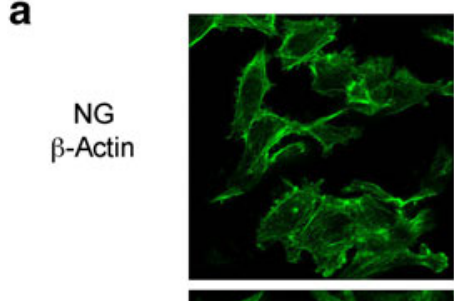

NG

RAGE
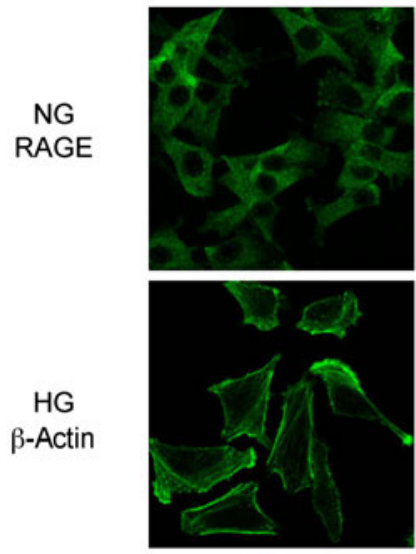

HG

$\beta$-Actin

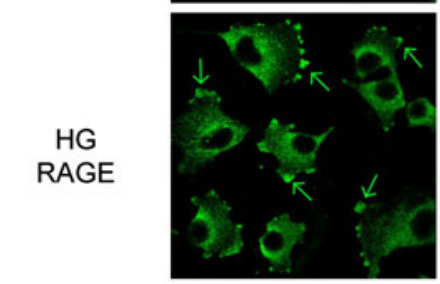

b
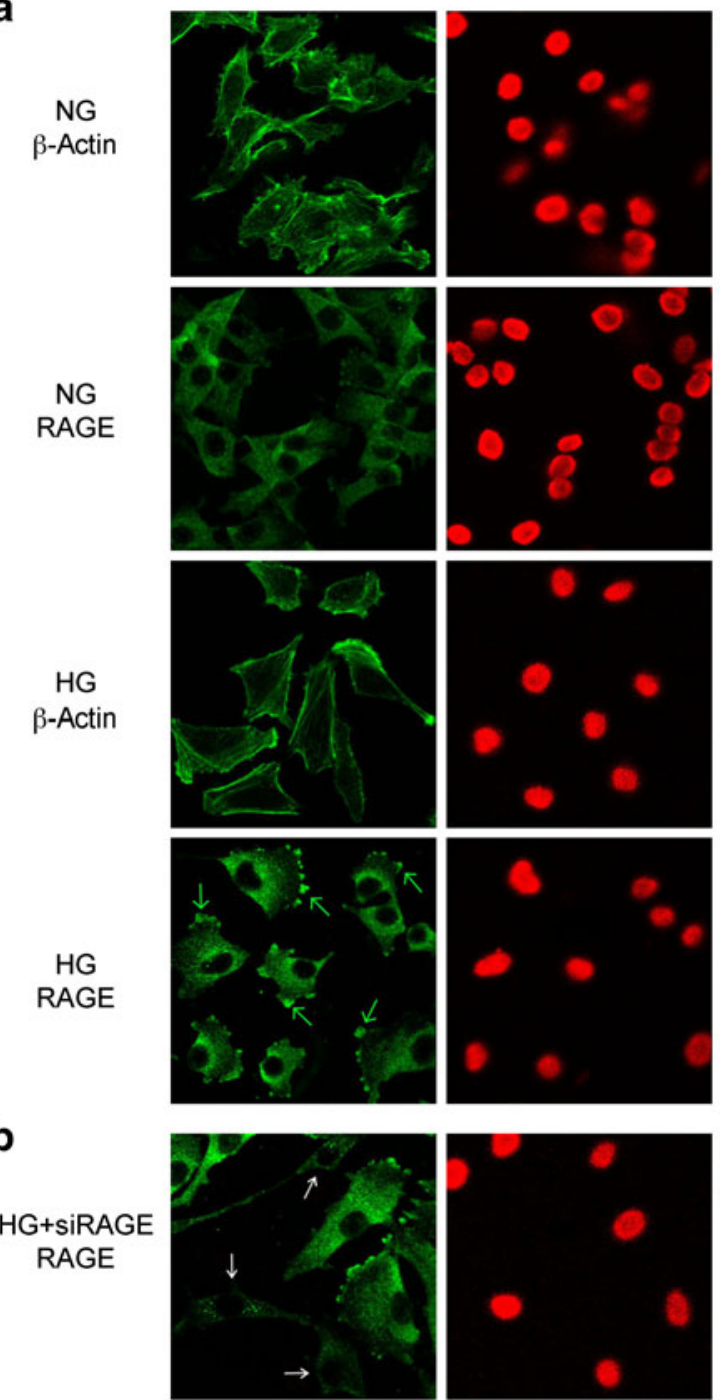

C
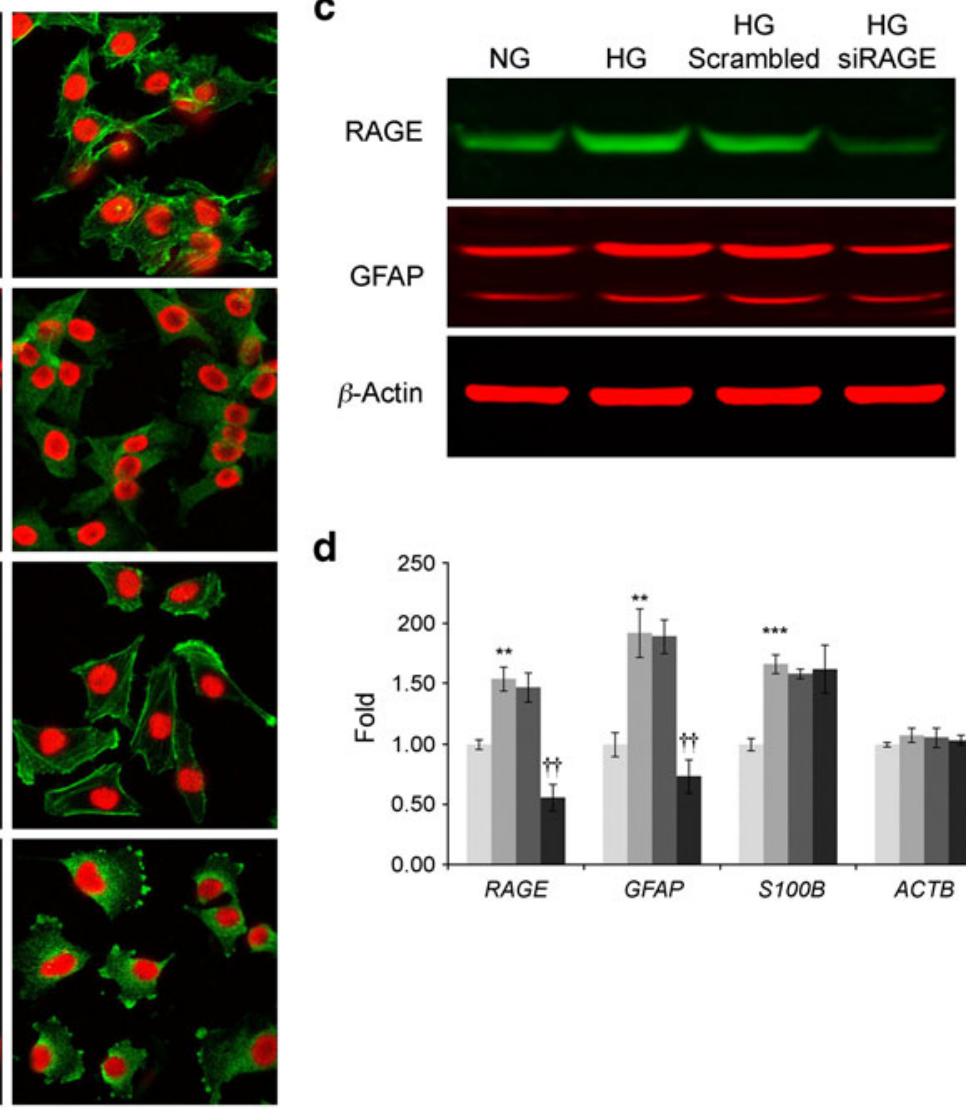

d

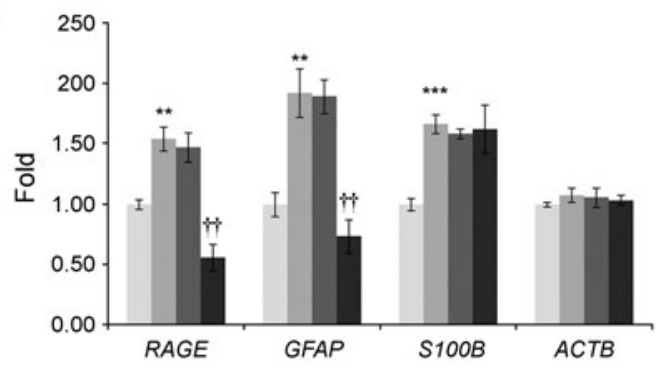

Fig. 2 RAGE levels are increased in Müller cell line cultured in high glucose in vitro. a MIO-M1 cells were grown in $5.5 \mathrm{mmol} / 1$ (normal glucose [NG]) or $25 \mathrm{mmol} / \mathrm{l}$ (high glucose [HG]) glucose containing DMEM for $48 \mathrm{~h}$. RAGE levels were determined by immunofluorescence using anti-RAGE antibody. Mouse anti- $\beta$-actin antibodies were employed as a positive control. Green arrows, pseudopodia of MIOM1 cells. b High glucose-cultured MIO-M1 cells were transfected with RAGE siRNA. At $48 \mathrm{~h}$ post transfection, cells were stained with anti-RAGE antibody. White arrows, Rage knockdown cells. c MIOM1 cells grown in normal glucose medium were transfected with scrambled control siRNA or RAGE-specific siRNA. At $24 \mathrm{~h}$ post transfection, cells were cultured in normal glucose or high glucose medium for another $48 \mathrm{~h}$. The protein levels of RAGE, GFAP and $\beta$ actin were detected by western blotting. d The mRNA levels of $R A G E, G F A P, S 100 B$ and $\beta$-actin $(A C T B)$ were determined by quantitative RT-PCR. Data are means \pm SE of triplicate cultures and are representative of three independent experiments. ${ }^{* *} p<0.01$ and $* * * p<0.001$ for S100B-treated vs PBS-treated cells; ${ }^{\dagger \dagger} p<0.01$ for RAGE siRNA vs scrambled RNA transfected cells. Light grey bars, normal glucose; medium grey bars, high glucose; dark grey bars, scrambled control siRNA with high glucose; black bars, RAGEspecific siRNA with high glucose induced after $24 \mathrm{~h}$ of high glucose exposure, also peaking at $48 \mathrm{~h}(p<0.01$ and $p<0.001$, respectively; Fig. 6$)$.

Consistent with the above data, VEGF peptide secretion was increased by exposure to high glucose $(p<0.001)$. Upon S100B exposure this response was significantly enhanced (Fig. 7a). To determine whether RAGE is central to high glucose and S100B-induced VEGF secretion, MIOM1 cells were transfected with RAGE siRNA or scrambled siRNA control, and exposed to normal or high glucose conditions. Loss of RAGE production in Müller glia resulted in inhibition of VEGF secretion following high glucose and S100B exposure (Fig. 7b).

An alternative approach to regulating receptor bioactivity is through blockade of ligand binding. It has been previously demonstrated that sRAGE is an effective inhibitor of RAGE-mediated responses [31]. We purified sRAGE as a GST-fusion protein from E. coli (Fig. 8a). In comparison with GST control protein, this protein pre- 
a

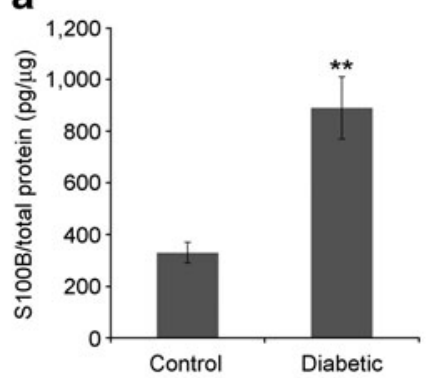

b

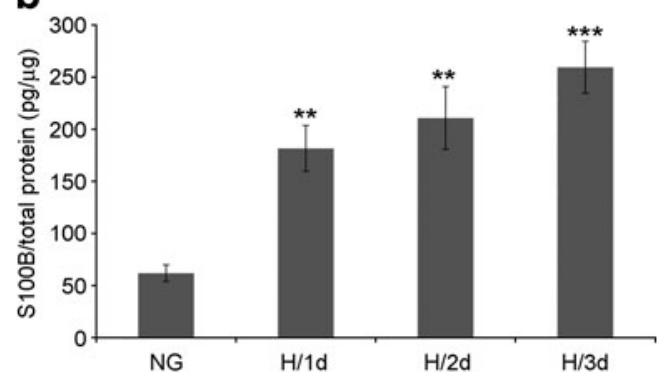

Fig. 3 S100B production is induced in diabetic retina and Müller glia exposed to high glucose. a S100B levels were determined by ELISA using $10 \mu \mathrm{g}$ of the total retinal protein extracted from non-diabetic or diabetic rat retina. ${ }^{*} p<0.01$ for diabetic rat retina vs non-diabetic rat retina. b MIO-M1 cells were maintained in high glucose medium for 1 to 3 days $(\mathrm{H} / 1 \mathrm{~d}, \mathrm{H} / 2 \mathrm{~d}, \mathrm{H} / 3 \mathrm{~d}$ respectively; $\mathrm{NG}$, normal glucose). $\mathrm{S} 100 \mathrm{~B}$ protein levels were measured by ELISA assay using $100 \mu \mathrm{g}$ of the total cell protein. Data are means \pm SE of triplicate cultures and are representative of three independent experiments. $* * p<0.01$ and $* * * p<0.001$ for high glucose-cultured MIO-M1 cells vs normal glucose-cultured MIO-M1 cells vented activation of the MAPK signalling pathway, as evidenced by inhibition of S100B-induced phosphorylation of p44/42 (Fig. 8b). High glucose- and S100B-induced VEGF secretion was likewise prevented by sRAGE $(p<$ 0.001; Fig. 8c).

\section{Discussion}

RAGE is recognised as an important mediator of inflammation in atherosclerosis, Alzheimer's disease and diabetic complications $[20,32]$. The RAGE axis has not been as extensively studied in the retina as in other tissues. The current study has therefore dissected the role of this receptor in the context of diabetic retinopathy and with a particular focus on the Müller glia, which demonstrate stress responses to the diabetic milieu by releasing harmful metabolites and cytokines [2]. Our data show that several pathophysiological responses by Müller glia are regulated by RAGE and add further evidence that this receptor could play an important role in diabetic retinopathy.

AGEs are not the only ligand for RAGE. In fact, it has been debated that proteins such as S100B could be more relevant physiological agonists for this receptor [33, 34]. While AGEs have already been shown to be present in diabetic retina, the current study demonstrated that RAGE and the inflammation-linked protein S100B are significantly upregulated during hyperglycaemia. RAGE production is widespread, while the Müller glia and astrocytes appear to be the principal cells producing $\mathrm{S} 100 \mathrm{~B}$ in the central
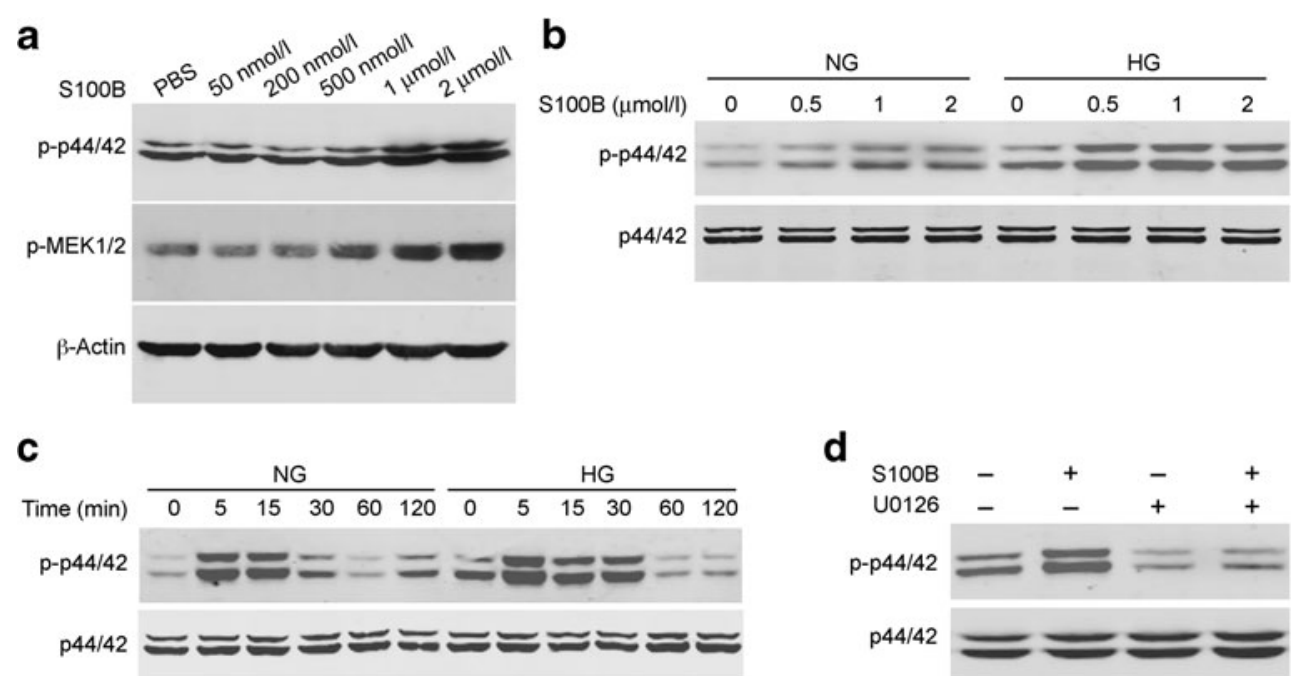

Fig. 4 S100B/RAGE activates MAPK signalling pathway. a MIOM1 cells were grown in 2\% FCS supplemented with normal glucose medium for 24 days followed by treatment with indicated amounts of S100B for $30 \mathrm{~min}$. Western blotting for phosphorylated (p)-p44/42 and MEK1/2 was performed. b MIO-M1 cells were grown in normal glucose (NG) or high glucose (HG) medium for $48 \mathrm{~h}$. Cells were then treated with indicated amounts of $\mathrm{S} 100 \mathrm{~B}$ for $30 \mathrm{~min}$ or with (c) $1 \mu \mathrm{mol} / 1$ of S100B for different time periods. Activation of MAPK pathway was evaluated by western blotting for p-p44/42 and p44/42. d MIO-M1 cells cultured in normal glucose were treated with $20 \mu \mathrm{mol} /$ $1 \mathrm{U} 0126$ for $1 \mathrm{~h}$ prior to treatment with $1 \mu \mathrm{mol} / \mathrm{l} \mathrm{S} 100 \mathrm{~B}$. Phosphorylation of $\mathrm{p} 44 / 42$ was detected by western blotting 

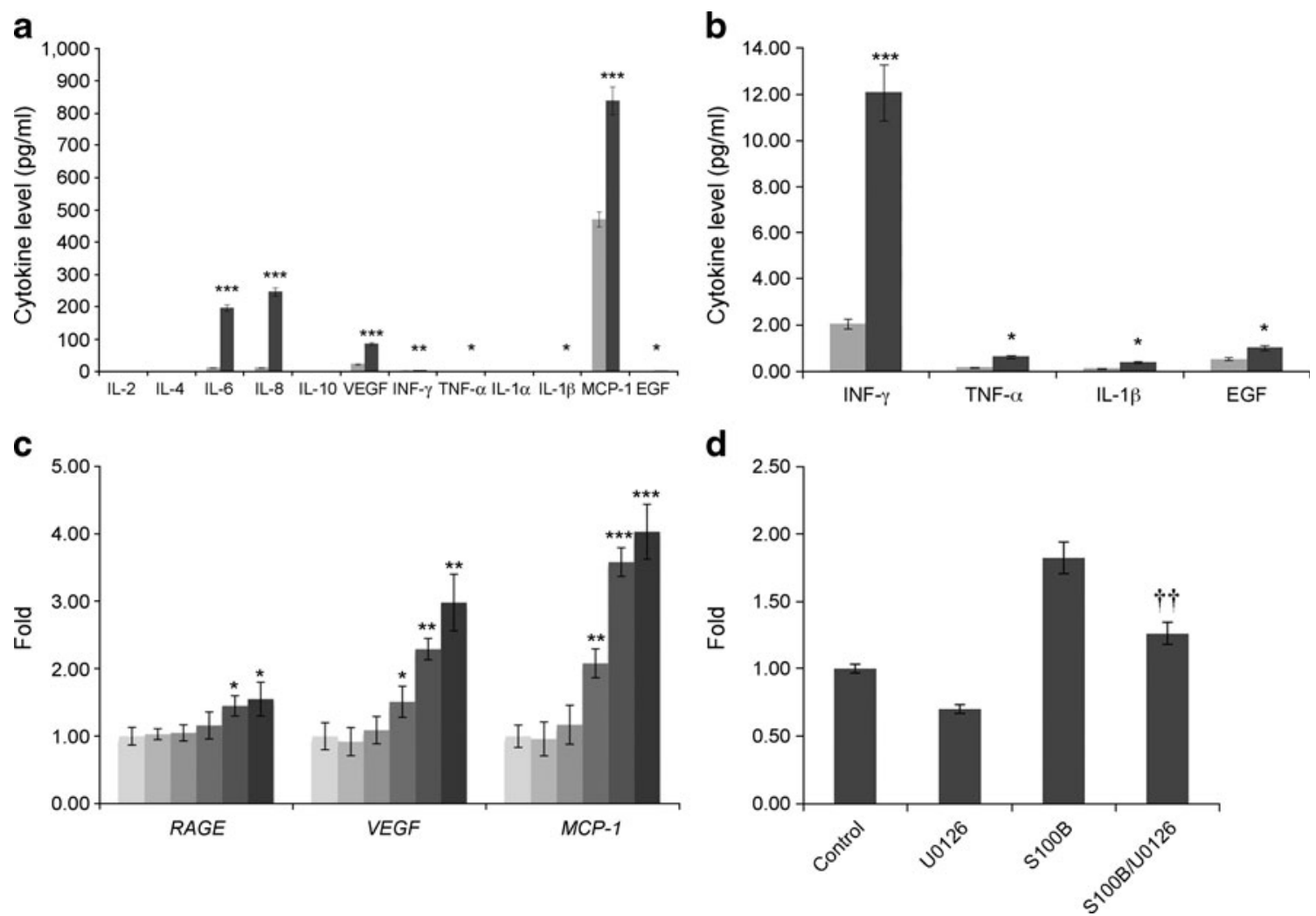

Fig. 5 S100B/RAGE induced proinflammatory cytokines in Müller cells. a, b MIO-M1 cells cultured in normal glucose medium were treated with $1 \mu \mathrm{mol} / 1 \mathrm{~S} 100 \mathrm{~B}$ (black bars) for $24 \mathrm{~h}$ for determination of the cytokine profile array indicated using the culture medium. Grey bars, PBS. c MIO-M1 cells as above were exposed to indicated amounts of $\mathrm{S} 100 \mathrm{~B}$ for $6 \mathrm{~h}$ to examine the mRNA levels of $R A G E, V E G F$ and $M C P-1$. Grey shades in rising intensity (light to dark): PBS,

nervous system [28]. While RAGE may be critical to endotheliopathy in diabetes [20], evaluation of this receptor and the downstream responses it controls in Müller glia has formed the focus of our investigation. We have demon-

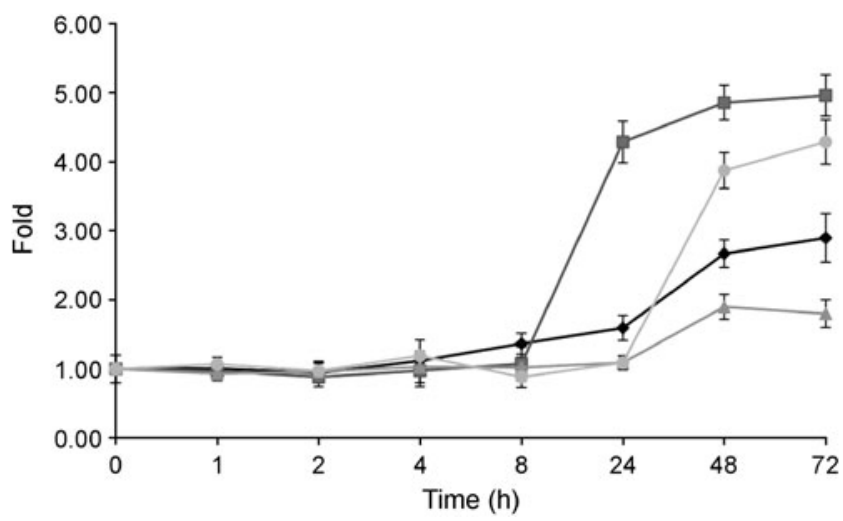

Fig. 6 High glucose induced RAGE and cytokine levels in Müller cells. MIO-M1 cells were culture in $25 \mathrm{mmol} / \mathrm{l}$ glucose-containing medium for different time periods. Expression of RAGE (black symbols), $S 100 B$ (grey squares), $V E G F$ (grey triangles) and $M C P-1$ (grey circles) was measured by quantitative RT-PCR. Data are means \pm SE of triplicate cultures and are representative of three independent experiments
$50 \mathrm{nmol} / \mathrm{l}, 200 \mathrm{nmol} / \mathrm{l}, 500 \mathrm{nmol} / \mathrm{l}, 1 \mu \mathrm{mol} / \mathrm{l}$ and $2 \mu \mathrm{mol} / \mathrm{l}$. d MIO-M1 cells as above were treated with $20 \mu \mathrm{mol} / 1 \mathrm{U} 0126$ for $1 \mathrm{~h}$ prior to treatment with $1 \mu \mathrm{mol} / 1 \mathrm{~S} 100 \mathrm{~B}$ for $24 \mathrm{~h}$, followed by quantitative RTPCR for $V E G F$. Data are means $\pm \mathrm{SE}$ of triplicate cultures and are representative of three independent experiments. ${ }^{*} p<0.05,{ }^{*} p<0.01$ and $* * * p<0.001$ for S100B-treated vs PBS-treated cells; ${ }^{\dagger \dagger} p<0.01$ for U0126+S100B-treated vs cells treated only with S100B

strated in vivo and in vitro that RAGE is associated with GFAP, a structural protein indicative of a general stress response by Müller glia [35]. Reduction of RAGE levels effectively prevents high glucose-induced upregulation of GFAP in vitro, although it remains to be determined whether this also occurs in an in vivo setting.

$\mathrm{S} 100 \mathrm{~B}$ is a member of a family of $\mathrm{Ca}^{2+}$-binding proteins of the EF-hand type [36] and is produced by various cell types including astrocytes, maturing oligodendrocytes, neural progenitor cells, Langerhans cells, dendritic cells and myoblasts [37]. S100B is specifically found in several cell types in bovine retina, including photoreceptors and Müller glial processes, in which S100B-stimulated membrane-bound guanylate cyclase activity can be induced [38]. GFAP and S100B content has been reported to be reduced in glia cultured in high glucose medium [39]. However, it was also demonstrated that GFAP and S100B are enhanced in the central nervous system during experimental diabetes mellitus [40]. These contradictory data encouraged us to verify the levels and molecular function of S100B in the diabetic retina, not least because it is a recognised ligand for RAGE. Our data reveal that 

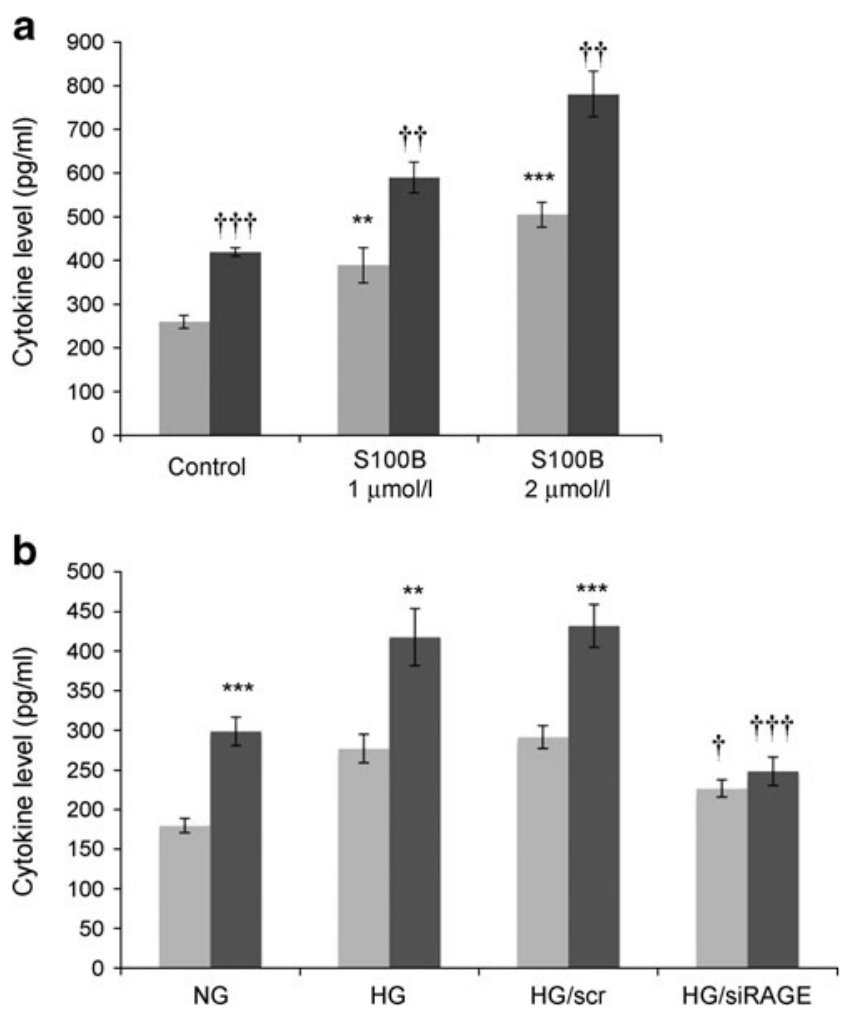

Fig. 7 High glucose hypersensitised Müller cells for RAGE induction. a MIO-M1 cells were cultured in normal glucose (NG, grey bars) or high glucose (HG, black bars) medium, and treated with $1 \mu \mathrm{mol} /$ 1 S100B. VEGF secretion was detected by ELISA. ${ }^{* *} p<0.01$ and $*^{* *} p<0.001$ for S100B-treated vs PBS-treated cells; $\dagger \dagger p<0.01$ and ${ }^{\dagger \dagger} p<0.001$ for cells cultured in high glucose medium vs culture in normal glucose. b MIO-M1 cells transfected with scrambled siRNA (scr) or RAGE siRNA (siRAGE) were maintained in normal or high glucose medium for $48 \mathrm{~h}$, followed by S100B treatment (black bars; control: grey bars) for another $24 \mathrm{~h}$. VEGF secretion was detected by ELISA. ${ }^{* *} p<0.01$ and ${ }^{* * *} p<0.001$ for S100B-treated vs PBS-treated cells; ${ }^{\dagger} p<0.05$ and ${ }^{\dagger \dagger} p<0.001$ for siRAGE- vs scrambled RNAtransfected cells. Data are means $\pm \mathrm{SE}$ of triplicate cultures and are representative of three independent experiments

a GST GST-SRAGE

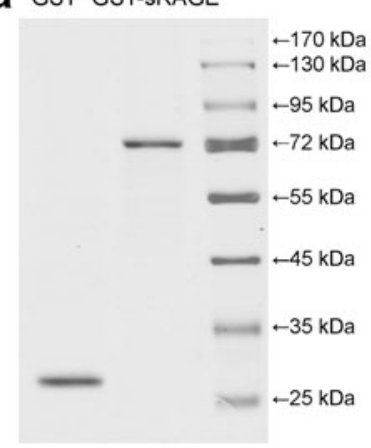

b

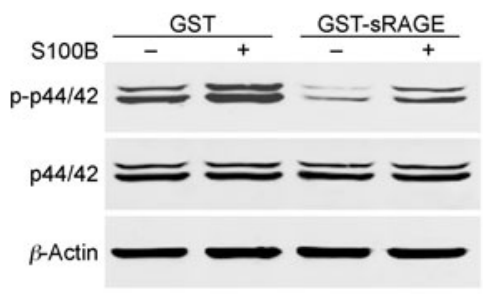

retinal GFAP and $\mathrm{S} 100 \mathrm{~B}$ are significantly induced by diabetes and co-localise with RAGE in Müller glia. Furthermore, S100B and RAGE are upregulated in Müller glia exposed in vitro to high glucose. Knockdown of S100B in MIO-M1 cells results in disassembly of stress fibres, cortical localisation of F-actin, time-dependent acquisition of a stellate morphology and a reduced migratory capacity [28]. We found that hyperglycaemia not only induced activity of the S100B/RAGE axis, but also morphological changes to cultured Müller glia as characterised by the appearance of increased numbers of pseudopodia where RAGE aggregated on the cells. Thus, overactivity of the S100B/RAGE axis in Müller glia could be linked to diabetes-induced gliosis.

Although many kinases of the MAPK cascade are activated by S100B-RAGE in HEK293T cells [41], RAGE-ligand binding in Müller glia induced p44/42, but not p38 and JNK (data not shown). This suggests that S100B-RAGE functions via different pathways in different cell types. RAGE activation is likely to be related to proinflammatory responses by Müller glia in diabetic retina $[9,12,42]$. However, it remains unknown whether RAGE could also be involved in pathogenic responses of more 'professional' inflammatory mediators in the diabetic retina, such as microglia and infiltrating monocytes [11, 43]. This investigation is ongoing in our laboratory.

Increased levels of proinflammatory cytokines in the diabetic retina has been linked to hypoxia and inflammation, a neurophile response to which the Müller glia may be a key component [5]. In retinal endothelial cells, high glucose exposure or activation of RAGE by S100B has been shown to induce levels of inflammatory cytokines such as COX2, VEGF and ICAMI [44]. S100B is neuroprotective at nanomolar levels. However, it shows a neurotoxic effect at micromolar levels in a RAGE-

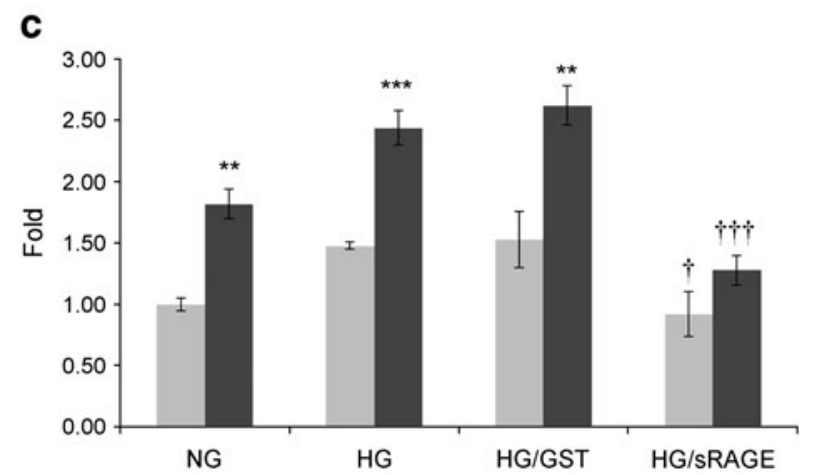

the same treatment for $24 \mathrm{~h}$ to detect VEGF secretion by ELISA. Black bars, S100B; grey bars, control. Data are means \pm SE of triplicate cultures and are representative of three independent experiments. $* * p<0.01$ and $* * * p<0.001$ for S100B-treated vs PBS-treated cells; ${ }^{\dagger} p<0.05$ and ${ }^{\dagger \dagger \dagger} p<0.001$ for sRAGE (GST-sRAGE)-treated vs GST control protein-treated cells 
dependent manner [36]. In the current study, S100B at doses lower than $500 \mathrm{nmol} / \mathrm{l}$ did not activate the MAPK pathway or induce cytokines in MIO-M1 cells. In contrast, S100B at over $500 \mathrm{nmol} / 1$ significantly induced Müller glia production of VEGF and MCP-1, which contributes to neovascularisation and attraction of microglia/monocytes to the deep retinal layers during diabetic retinopathy $[45,46]$. This may have been achieved via MEK1/2 and p44/42, as inhibition of MEK $1 / 2$ in our experiments attenuated S100B-induced VEGF production. Although RAGE might not be the sole receptor for S100B, the proinflammatory effect of S100B on Müller glia is largely due to RAGEmediated signalling as evidenced by failure of S100Binduced cytokine levels in $R A G E$ knockdown or in sRAGEtreated MIO-M1 cells. Such a finding suggests a central role for RAGE in hyperglycaemia and S100B-induced VEGF and MCP-1 upregulation. This is significant because RAGE blockade has the potential to regulate two important aspects of diabetic retinopathy, namely angiogenic/vasopermeability growth factor and pro-inflammatory cytokine release.

It would be interesting to determine whether the changes in retinal S100B levels reflect changes in the serum. Furthermore, the central role of RAGE in S100B-induced Müller glia activation in vivo has not been fully addressed in this study. Studies using specific RAGE blockade or the use of diabetic $R A G E^{-1-}$ mice could help build on the current data, establishing perhaps the link between the S100B-RAGE axis and the glial, neural and vascular degeneration known to occur in diabetic retinopathy.

In conclusion, we have demonstrated that hyperglycaemiainduced RAGE plays an important role in Müller glial activation and downstream cytokine production in the context of diabetic retinopathy. The RAGE axis could play an important role in the pathogenesis of diabetic retinopathy and agents capable of attenuating RAGE signalling could prove useful in preventing this complication.

Acknowledgements This work is supported by the Juvenile Diabetes Research Foundation, D.E.L. Northern Ireland and Fight for Sight (UK).

Duality of interest The authors declare that there is no duality of interest associated with this manuscript.

\section{References}

1. Curtis TM, Gardiner TA, Stitt AW (2009) Microvascular lesions of diabetic retinopathy: clues towards understanding pathogenesis? Eye (Lond) 23:1496-1508

2. Bringmann A, Pannicke T, Grosche J et al (2006) Muller cells in the healthy and diseased retina. Prog Retin Eye Res 25:397-424
3. Gerhardinger C, Brown LF, Roy S, Mizutani M, Zucker CL, Lorenzi M (1998) Expression of vascular endothelial growth factor in the human retina and in nonproliferative diabetic retinopathy. Am J Pathol 152:1453-1462

4. Lieth E, Barber AJ, Xu B et al (1998) Glial reactivity and impaired glutamate metabolism in short-term experimental diabetic retinopathy. Penn State Retina Research Group. Diabetes $47: 815-820$

5. Bai Y, Ma JX, Guo J et al (2009) Muller cell-derived VEGF is a significant contributor to retinal neovascularization. J Pathol 219:446-454

6. Pannicke T, Iandiev I, Wurm A et al (2006) Diabetes alters osmotic swelling characteristics and membrane conductance of glial cells in rat retina. Diabetes 55:633-639

7. Joussen AM, Poulaki V, Le ML et al (2004) A central role for inflammation in the pathogenesis of diabetic retinopathy. FASEB J 18:1450-1452

8. Gerhardinger C, Dagher Z, Sebastiani P, Park YS, Lorenzi M (2009) The transforming growth factor-beta pathway is a common target of drugs that prevent experimental diabetic retinopathy. Diabetes 58:1659-1667

9. Brucklacher RM, Patel KM, Van Guilder HD et al (2008) Whole genome assessment of the retinal response to diabetes reveals a progressive neurovascular inflammatory response. BMC Med Genomics 1:26

10. Vincent AM, Perrone L, Sullivan KA et al (2007) Receptor for advanced glycation end products activation injures primary sensory neurons via oxidative stress. Endocrinology 148:548-558

11. Rungger-Brandle E, Dosso AA, Leuenberger PM (2000) Glial reactivity, an early feature of diabetic retinopathy. Invest Ophthalmol Vis Sci 41:1971-1980

12. Gerhardinger C, Costa MB, Coulombe MC, Toth I, Hoehn T, Grosu P (2005) Expression of acute-phase response proteins in retinal Muller cells in diabetes. Invest Ophthalmol Vis Sci 46:349-357

13. Schmidt AM, Hofmann M, Taguchi A, Yan SD, Stern DM (2000) RAGE: a multiligand receptor contributing to the cellular response in diabetic vasculopathy and inflammation. Semin Thromb Hemost 26:485-493

14. Yan SF, Ramasamy R, Schmidt AM (2010) The RAGE axis: a fundamental mechanism signaling danger to the vulnerable vasculature. Circ Res 106:842-853

15. Neeper M, Schmidt AM, Brett J et al (1992) Cloning and expression of a cell surface receptor for advanced glycosylation end products of proteins. J Biol Chem 267:14998-15004

16. Yeh CH, Sturgis L, Haidacher J et al (2001) Requirement for $\mathrm{p} 38$ and $\mathrm{p} 44 / \mathrm{p} 42$ mitogen-activated protein kinases in RAGEmediated nuclear factor-kappaB transcriptional activation and cytokine secretion. Diabetes 50:1495-1504

17. Huttunen HJ, Fages C, Rauvala H (1999) Receptor for advanced glycation end products (RAGE)-mediated neurite outgrowth and activation of NF-kappaB require the cytoplasmic domain of the receptor but different downstream signaling pathways. J Biol Chem 274:19919-19924

18. Hirose A, Tanikawa T, Mori H, Okada Y, Tanaka Y (2010) Advanced glycation end products increase endothelial permeability through the RAGE/Rho signaling pathway. FEBS Lett 584:6166

19. Huang JS, Guh JY, Chen HC, Hung WC, Lai YH, Chuang LY (2001) Role of receptor for advanced glycation end-product (RAGE) and the JAK/STAT-signaling pathway in AGE-induced collagen production in NRK-49F cells. J Cell Biochem 81:102113

20. Ramasamy R, Yan SF, Schmidt AM (2005) The RAGE axis and endothelial dysfunction: maladaptive roles in the diabetic vasculature and beyond. Trends Cardiovasc Med 15:237-243 
21. Barile GR, Pachydaki SI, Tari SR et al (2005) The RAGE axis in early diabetic retinopathy. Invest Ophthalmol Vis Sci 46:29162924

22. Soulis T, Thallas V, Youssef S et al (1997) Advanced glycation end products and their receptors co-localise in rat organs susceptible to diabetic microvascular injury. Diabetologia 40:619-628

23. Hammes HP, Hoerauf H, Alt A et al (1999) N(epsilon)(carboxymethyl)lysin and the AGE receptor RAGE colocalize in agerelated macular degeneration. Invest Ophthalmol Vis Sci 40:1855-1859

24. Stitt A, Gardiner TA, Alderson NL et al (2002) The AGE inhibitor pyridoxamine inhibits development of retinopathy in experimental diabetes. Diabetes 51:2826-2832

25. Pachydaki SI, Tari SR, Lee SE et al (2006) Upregulation of RAGE and its ligands in proliferative retinal disease. Exp Eye Res 82:807-815

26. Yao D, Brownlee M (2010) Hyperglycemia-induced reactive oxygen species increase expression of the receptor for advanced glycation end products (RAGE) and RAGE ligands. Diabetes 59:249-255

27. Limb GA, Salt TE, Munro PM, Moss SE, Khaw PT (2002) In vitro characterization of a spontaneously immortalized human Muller cell line (MIO-M1). Invest Ophthalmol Vis Sci 43:864869

28. Brozzi F, Arcuri C, Giambanco I, Donato R (2009) S100B protein regulates astrocyte shape and migration via interaction with Src kinase: implications for astrocyte development, activation, and tumor growth. J Biol Chem 284:8797-8811

29. Howes KA, Liu Y, Dunaief JL et al (2004) Receptor for advanced glycation end products and age-related macular degeneration. Invest Ophthalmol Vis Sci 45:3713-3720

30. Busik JV, Mohr S, Grant MB (2008) Hyperglycemia-induced reactive oxygen species toxicity to endothelial cells is dependent on paracrine mediators. Diabetes 57:1952-1965

31. Bucciarelli LG, Wendt T, Qu W et al (2002) RAGE blockade stabilizes established atherosclerosis in diabetic apolipoprotein Enull mice. Circulation 106:2827-2835

32. Yan SF, Ramasamy R, Schmidt AM (2008) Mechanisms of disease: advanced glycation end-products and their receptor in inflammation and diabetes complications. Nat Clin Pract Endocrinol Metab 4:285-293

33. Thornalley PJ (2007) Dietary AGEs and ALEs and risk to human health by their interaction with the receptor for advanced glycation endproducts (RAGE) — an introduction. Mol Nutr Food Res 51:1107-1110

34. Ramasamy R, Yan SF, Schmidt AM (2007) Arguing for the motion: yes, RAGE is a receptor for advanced glycation endproducts. Mol Nutr Food Res 51:1111-1115

35. Izumi Y, Kirby CO, Benz AM, Olney JW, Zorumski CF (1999) Muller cell swelling, glutamate uptake, and excitotoxic neurodegeneration in the isolated rat retina. Glia 25:379-389

36. Donato R (2001) S100: a multigenic family of calcium-modulated proteins of the EF-hand type with intracellular and extracellular functional roles. Int J Biochem Cell Biol 33:637-668

37. Donato R, Sorci G, Riuzzi F et al (2009) S100B's double life: intracellular regulator and extracellular signal. Biochim Biophys Acta 1793:1008-1022

38. Rambotti MG, Giambanco I, Spreca A, Donato R (1999) S100B and S100A1 proteins in bovine retina: their calcium-dependent stimulation of a membrane-bound guanylate cyclase activity as investigated by ultracytochemistry. Neuroscience 92:1089-1101

39. Nardin P, Tramontina F, Leite MC et al (2007) S100B content and secretion decrease in astrocytes cultured in high-glucose medium. Neurochem Int 50:774-782

40. Baydas G, Nedzvetskii VS, Tuzcu M, Yasar A, Kirichenko SV (2003) Increase of glial fibrillary acidic protein and S-100B in hippocampus and cortex of diabetic rats: effects of vitamin E. Eur J Pharmacol 462:67-71

41. Zong H, Madden A, Ward M, Mooney MH, Elliott CT, Stitt AW (2010) Homodimerization is essential for the receptor for advanced glycation end products (RAGE)-mediated signal transduction. J Biol Chem 285:23137-23146

42. Shelton MD, Kern TS, Mieyal JJ (2007) Glutaredoxin regulates nuclear factor kappa-B and intercellular adhesion molecule in Muller cells: model of diabetic retinopathy. J Biol Chem 282:12467-12474

43. Zeng HY, Green WR, Tso MO (2008) Microglial activation in human diabetic retinopathy. Arch Ophthalmol 126:227-232

44. Perrone L, Devi TS, Hosoya K, Terasaki T, Singh LP (2009) Thioredoxin interacting protein (TXNIP) induces inflammation through chromatin modification in retinal capillary endothelial cells under diabetic conditions. J Cell Physiol 221:262-272

45. Crawford TN, Alfaro DV 3rd, Kerrison JB, Jablon EP (2009) Diabetic retinopathy and angiogenesis. Curr Diabetes Rev 5:8-13

46. Nakazawa T, Hisatomi T, Nakazawa C et al (2007) Monocyte chemoattractant protein 1 mediates retinal detachment-induced photoreceptor apoptosis. Proc Natl Acad Sci USA 104:2425-2430 